\title{
Single-blind randomized clinical trial on the efficacy of an interdental cleaning device in orthodontic patients
}

\author{
Alessandro QUARANTA 1 *, Olivia MARCHISIO ${ }^{2}$, Orlando D'ISIDORO 1, 3 , \\ Anna-Maria GENOVESI 2 , Ugo COVANI 2
}

${ }^{1}$ Research Program in Oral Implantology and Periodontics, UWA Dental School, University of Western Australia, Nedlands, WA, Australia; ${ }^{2}$ Department of Dentistry, Tuscan Institute of Stomatology, Versilia General Hospital, Lido di Camaiore, Lucca, Italy; ${ }^{3}$ Private Practitioner, Silvi Marina, Teramo, Italy

*Corresponding author: Alessandro Quaranta, Discipline of Periodontics and Oral Implantology, UWA Dental School, University of Western Australia, 17 Monash Avenue, Nedlands, WA 6009, Australia. E-mail: alex.quaranta@uwa.edu.au

BACKGROUND: It is well-known that poor oral hygiene during orthodontic treatment may lead to development of gingivitis, probing pocket depth, hyperplastic tissue, decalcification, dental caries and white spot lesions on the coronal surfaces of teeth.

METHODS: Twenty-two patients with the following inclusion criteria were enrolled in the present study: fixed orthodontic treatment, age 12-18 years, systemically healthy, no history of periodontal treatment, periodontal health or gingivitis. The following clinical parameters were collected: periodontal screening and recording, plaque control record, and bleeding on probing. Oral hygiene instructions were given and a specifically designed technique for orthodontic patients was suggested. Complete information about the mechanical interdental device were also provided. Means and standard deviations or medians and interquartile ranges for each parameter were collected.

RESULTS: There was a statistically significant reduction in plaque control record on both sides. The reduction in the experimental group was significantly greater compared to controls after adjusting for baseline values. There was also a statistically significant reduction in bleeding on probing in the experiment group, but not in controls.

CONCLUSIONS: The combination of manual toothbrush and mechanical interdental device demonstrated a better plaque control and gingival inflammation levels in orthodontic patients compared to manual brushing alone.

(Cite this article as: Quaranta A, Marchisio O, D'Isidoro O, Genovesi AM, Covani U. Single-blind randomized clinical trial on the efficacy of an interdental cleaning device in orthodontic patients. Minerva Stomatol 2018;67: S0026-4970.18.04104-3)

Key words: Oral hygiene - Dental plaque - Home care dental devices - Orthodontics.

$\mathrm{I}_{\mathrm{i}}^{\mathrm{t}}$ is well-known that poor oral hygiene during orthodontic treatment leads to develop gingivitis, probing pocket depth, hyperplastic tissue, decalcification, dental caries and white spot lesions on the coronal surfaces of teeth. ${ }^{1-3}$

The earliest sigs of enamel caries are the white spot lesions (WSL). ${ }^{4}$ Arches, tubes, ligatures, brackets facilitate plaque retention increasing microbial load and decreasing $\mathrm{pH}$ with the onset of demineralization in subsurface of the enamel.5 As demonstrated by O'Reilly et al. ${ }^{6}$ and Ogaard et al., ${ }^{7}$ development of clinically visible WSL in orthodontic patients can occur within 4 weeks or sooner.

In a study by Maxfield et al., it is shown that this may result in an esthetic damage, influencing the patient's relations. Patients, parents, orthodontists, and general dentists had similar 
perceptions regarding the significance, prevention, and treatment of white spot lesions. All groups indicated that patients were the most responsible for the prevention of white spot lesions. Communication among patients, parents, orthodontists, and general dentists needed to be improved to decrease the incidence of white spot lesions in the orthodontic population. ${ }^{8}$

Kim et al. demonstrated how orthodontic treatment may lead to gingival inflammation and how orthodontic appliances affect the subgingival microbial composition even during the early period of orthodontic treatment, increasing the prevalence of periodontal bacteria ${ }^{9}$. Among these pathogens we can include including $F$. nucleatum and Porphyromonas gingivalis. ${ }^{10,11}$

Tanner et al. characterized the microbiota of WSL, accounting for confounding due to gingivitis. Sixty children aged between 10 and 19 years with fixed appliances were enrolled and the half of them had WSL. Plaque samples were assayed by a $16 \mathrm{~S}$ rRNA-based microarray and by PCR. Mean gingival index was positively associated with WSL $(\mathrm{P}=0.018)$. Taxa associated with gingivitis by microarray included: Gemella sanguinis, Actinomyces spp., Prevotella cluster IV and Streptococcus spp. HOT 071/070; Many taxa showed a stronger association with gingivitis than with WSL. 12

Recent studies show that there is not a significant association between type of malocclusion and gingival biotype. There is however a prevalence of thick gingival biotype in patient with class II malocclusion and a slight prevalence of thin gingival biotype in patient with class I malocclusion. ${ }^{13}$

An in-vivo study was carried out in 2011 to assess the effect of sonic device on interproximal plaque and gingivitis when used in addition to manual toothbrushing. One hundred and forty-eight adults (98 females, 50 males; mean age 39.5 years) with moderate gingivitis participated in this single-blind, four-week, parallel, randomized controlled clinical trial. Subjects were randomized either to a manual toothbrush (two minutes, twice a day) or to a manual toothbrush plus sonic device (once daily, evening). Changes in gingival inflam- mation parameters were recorded, and plaque samples were analyzed the residual protein concentration. The Authors concluded that the sonic interdental cleaning, when used in addition to a manual toothbrush, provided significantly greater reductions in gingivitis and bleeding sites $(\mathrm{P}<0.01)$ than a manual toothbrush alone. After four weeks, it reduced gingivitis by $33 \%$ more, gingival bleeding by $75 \%$ more and the number of bleeding sites by $86 \%$ more than a manual toothbrush alone. Interproximal plaque evaluated after a single use showed that interdental sonic devices removed significantly more plaque than a manual toothbrush alone $(\mathrm{P}<0.01)$. Both products were safe to use. This kind of technology, when used in addition to manual brushing, removed significantly more interproximal plaque and resulted in significantly greater reductions of gingivitis after two weeks and four weeks of use, compared to manual brushing alone. ${ }^{14}$

The aim of this study is to assess the effect of a mechanical interdental cleaning device combined with manual toothbrush in the improvement of self-performed plaque control and gingival health in patients undergoing orthodontic treatment.

\section{Materials and methods}

This paper was carried out according to WMA Declaration of Helsinki, recently reviewed at 64th WMA General Assembly in Fortaleza, Brazil, in October 2013.

According the CONSORT guidelines published by the Lancet on March 24th, 2010, and according to the CONSORT flow diagram of the progress through the phases of a parallel randomized trial of two groups, we enriched the materials and method section. First of all, the assessment for eligibility was carried out, and no exclusion was made (all participants fulfilled the inclusion criteria and no declinations were observed).

Twenty-two patients under orthodontic treatment in the Clinical Faculties of the Dental School of the Marche Polytechnic University were enrolled in January 2014 and joined the clinical study between February 2014 and 
May 2014. Each patient contributed to the study in a split mouth fashion (one side test and one side control). Patients have given their informed consent for participation in the research study. The study was carried out at the University Periodontal Clinic.

The patients enrolled fulfilled the following inclusion criteria: fixed orthodontic treatment in both jaws, age between 12 and 18, and a Periodontal Screening and Recording of the American Academy of Periodontology (PSR) Score of 1 or 2. Patients affected by periodontitis, patients younger than 12 or older than 18 , and psychophysically-compromised patients were excluded.

For each patient a clinical record consisting of medical history, plaque control record (PCR), ${ }^{15}$ bleeding on probing (BOP), ${ }^{16}$ and intraoral photos was collected.

After obtaining informed consent, each patient's side was randomly assigned to two groups: an experimental group, using medium orthodontic toothbrush and Sonicare Air Floss ${ }^{\circledR}$, and a control group, using medium orthodontic toothbrush only.

The patients were randomly allocated using a free online randomizer (www.randomization.com), which prints simple lists of random allocations.

The study was divided in three sessions, each taking place 15 days after the previous one, which were carried out by two experienced operators. Discussion and consensus were used to resolve disagreements about the clinical parameters collection.

In cases where mutual agreement between the examiners could not be reached, a third Author assessed the study and a final decision was taken.

\section{First session}

During the first session, a detailed medical history was compiled. Subsequently, PSR, BOP, and PCR indexes were collected. In addition, a photo status (consisting of intra-oral frontal, lateral, lingual and palatal photographs) before and after plaque detection was performed. The first visit was performed by the first clinician. At the end of the first session, oral hygiene devices were given, and motivation and instructions were provided, depending on the group to which patients were assigned. The following instructions were provided by two senior dental hygiene students:

- orthodontic toothbrush and brushing method: brush teeth twice a day for at least two minutes with orthodontic toothbrush and toothpaste. Modified Bass technique for buccal surfaces followed by short horizontal movements to clean the brackets; modified Bass technique for lingual/palate surfaces;

- Sonicare Air Floss ${ }^{\circledR}$ : place the tip of the device in the interdental space and press the activation button; move the tip to the next dental space and repeat the operation, without neglecting the hard-to-reach spots around the second molars.

\section{Second session}

After 15 days, the second session was carried out by the third author and the same clinical investigators of the first session. Data on patient comfort when using Sonicare Air Floss were also collected.

\section{Third session}

During the third and last session, that was carried out one month later, the following indexes were collected again by the first author: PSR, BOP, PCR, and photographic status (consisting of frontal, lateral, lingual and palatal photographs) before and after plaque detection. In addition, information on patient comfort with the use of Sonicare Air Floss was collected.

\section{Statistical analysis}

The primary outcome was the plaque index in $\%$, while the secondary outcome was the BOP reduction. A statistical power of $80 \%$ and a C.I of $95 \%$ were considered statistically significant.

The sample size was calculated as follows using SPSS Statistics software: 
- first variable: supragingival plaque reduction;

$-Z$ tests: proportions: difference between two independent proportions

- analysis: a priori: computer; required sample size.

A total size of 34 samples (group 1, N. $=17$, group $2, \mathrm{~N} .=17$ ) corresponds to a statistical power of $91 \%(0.9073357)$ with a critical $Z$ value of 1.9599640 .

\section{Results}

\section{PCR}

There was a statistically significant reduction in both sides (control $-5.5,95 \% \mathrm{CI}-9.2$ to $-1.7, p=0.006$; treatment $-12.9,95 \%$ CI -15.8 to $9.9, \mathrm{P}<0.001$ ) with the reduction on the treatment side statistically significantly greater compared to the control side after adjusting for baseline values $(-7.6,95 \% \mathrm{CI}:-11.1$ to -4.0 , $\mathrm{P}<0.001)$ (Table I).

\section{BOP score}

Medians and inter-quartile ranges shown due to skewed values, although model residuals were acceptable without transformation (Table II).

There was a statistically significant reduction on the treatment side $(-3.4,95 \% \mathrm{CI}:-5.0$ to $-1.7, \mathrm{P}<0.001)$ but not the control side $(-0.8$, 95\% CI: -2.3 to $0.7, \mathrm{P}=0.272$ ).

The reduction on the treatment side was statistically significantly greater compared to the control side after adjusting for baseline values $(-1.8,95 \%$ CI: -3.0 to $-0.6, \mathrm{P}=0.004)$.

The treatment side show a greater reduction of clinical parameters compared to the control side. The mechanical device has a greater im-

TABLE I.-Plaque control record score.

\begin{tabular}{lcc}
\hline & Control group (N.=20) & Experimental group (N.=20) \\
\hline Baseline & $54.7 \pm 14.1$ & $53.9 \pm 13.7$ \\
Follow-up & $49.3 \pm 14.0$ & $41.0 \pm 12.1$ \\
Change & $-5.5 \pm 8.4$ & $-12.9 \pm 6.7$ \\
\hline Data presented as mean \pm SD. &
\end{tabular}

pact on plaque control record. BoP does not reveal a statistically significant reduction on the control side.

\section{Discussion}

The orthodontic forces and movements induce hard and soft tissues responses, as well described by Antoun et al. In general, the controlled movement of teeth seems to have a positive, but highly variable effect on the supporting tissues. ${ }^{17}$ Davies et al. note that inflammation and hyperplasia can affect even patients with a good oral hygiene. This gingival enlargement explains some of the reported increases in probing depths, which are not related to attachment loss and is resolved as early as 48 hours after band removal, suggesting that the appliances themselves have an influence on periodontal health unrelated to plaqueinduced periodontal disease. ${ }^{18}$

However, as confirmed by the literature, poor oral hygiene and plaque accumulation, worsens the periodontal conditions and the long-term success of the orthodontic treatment. This study shows that a combination of manual toothbrush and interdental device demonstrated a better control of plaque and gingival inflammation levels compared to manual brushing alone in patients undergoing orthodontic treatment. Our results are similar to those obtained by de Jager et al. ${ }^{14}$

The first factor to consider is that patients show a better compliance in the use of Sonicare AirFloss ${ }^{\circledR}$. Krell et al. in a study about the device use and compliance, showed that after one month of use, irregular flossers (people who floss from once a month to three times a week) changed their habits using the mechanical interdental device on an average of 1.3

TABLE II.-Bleeding on probing score.

\begin{tabular}{lcc}
\hline & Control group $(\mathrm{N} .=20)$ & Experimental group $(\mathrm{N} .=20)$ \\
\hline Baseline & $3.5(7.0)$ & $5.0(5.0)$ \\
Follow-up & $3.0(6.0)$ & $0.5(6.0)$ \\
Change & $-0.8 \pm 3.4$ & $-3.4 \pm 3.8$ \\
\hline
\end{tabular}

Data presented as median (IQR) or as mean \pm SD. 
times per day. Moreover $96.1 \%$ of participants used the device four or more days per week. ${ }^{19}$ In a similar study, $86 \%$ of participants reported the mechanical interdental device easier to use than floss, and $69 \%$ reported it easier to use than an oral irrigator. About 78\% reported the AirFloss $^{\circledR}$ is more gentle on teeth and gums than string floss; $81 \%$ reported that it was easier to use in the back of the mouth compared to a string floss. ${ }^{20}$

We should also consider that the Hawthorn's effect could affect this considerations about compliance. Behavioral change during a study can often be explained by the Hawthorn effect. Patients who believe they are participating in a study generally change behavior when requested; patients who do not know to be involved in a study do not generally show a great a change in behavior. ${ }^{21}$

A third factor to focus on is that similar results find general agreement in the literature. A study by Lanese et al. compared conventional toothbrush alone (control), electric toothbrush alone, conventional toothbrushing with oral irrigation device, powered toothbrush with oral irrigation device, as home use oral hygiene methods in adult fixed orthodontic patients. The use of oral irrigation with or without a power toothbrush yielded significant reduction in plaque, gingival inflammation, and a tendency for reduced BOP. ${ }^{22}$ Sharma et al. selected 82 subjects for a randomized, fourweek, single-blind, two-group parallel clinical study. Subjects were randomly assigned to one of two groups: Waterpik ${ }^{\circledR}$ Water Flosser plus manual toothbrushing or Sonicare ${ }^{\circledR}$ AirFloss plus manual toothbrushing. Oral irrigation with the specialized orthodontic tip was shown to have a 3.76-fold the reduction in plaque index as flossing and a 5.83-fold the reduction in plaque index as the toothbrushing alone. ${ }^{23}$ A recent meta-analysis confirms that highfrequency, high-amplitude sonic powered toothbrushes decreased plaque and gingivitis significantly more effectively than manual toothbrushes in everyday use in studies lasting up to three months ${ }^{24}$.

A fourth factor to investigate in the future is the possibility to extend this device to non- orthodontic population and use it in daily hygiene. A study by Walsh et al. compared the effects of four oral hygiene methods (manual toothbrushing, power toothbrushing, manual toothbrushing plus irrigation, and power toothbrushing plus irrigation) on plaque and periodontal disease on 108 subjects. Results showed that all the oral hygiene methods were equally effective in reducing plaque and stain accumulation, gingival bleeding, bleeding to probing ratio and the rate of pockets $4 \mathrm{~mm}$ or deeper. None of the oral hygiene methods was associated with injury to soft or hard tissues. ${ }^{25}$ The effectiveness in reducing the inflammation clinical parameters is confirmed by another work. ${ }^{26}$

A discording result is shown by Barnes et al. Comparing manual and sonic toothbrushing combined with flossing and a water jet found that groups with oral irrigation were significantly more effective in reducing bleeding, groups with oral irrigation had significantly lower gingival index on the facial surfaces of teeth, and only the combination of a sonic toothbrush with the oral irrigator had a significantly lower plaque index. ${ }^{5}$ In non-orthodontic patients, oral irrigation can significantly reduce $\mathrm{BI}$ and GI, but likely has minimal effect on PI. This results do not clearly affirm that Sonic technology must replace standard flossing to increase clinical parameters in non-orthodontic patients. ${ }^{27}$

All these considerations cannot underestimate the influence of some factors affecting the orthodontic and periodontal treatment outcomes. Indeed, the analysis of the literature, demonstrate that plaque control, however fundamental, is not the only variable to achieve a good periodontal stability. Other factors such as the kind of malocclusion, the gingival biotype, the severity of periodontal disease and the bone loss pattern must be considered. Depending on the diagnosed malocclusion, differing orthodontic strategies can be utilized to achieve optimal results. A careful assessment of the patients' overall biotype and the necessary tooth movement vectors for optimal results is therefore crucial to determine if surgical gingival augmentation is necessary prior 
to orthodontic tooth movement. ${ }^{28}$ Moreover, recent studies are focusing on the mechanobiology of the orthodontic movement. Biologically, not only the intensity of the force, but also its duration and the tissue response to the application of the same are important for tooth movement. In the light of this, it will possible to examine the concept of optimal orthodontic force, a determining factor for the success of orthodontic treatment. ${ }^{29}$ As a recent case report confirms, a correct position of the tooth, even in impacted canines, can be achieved 30 when all these factors are considered.

\section{Conclusions}

The sample size may be one of the limits of this study. Other studies available in the literature are performed on bigger samples (from 60 to 148). Moreover, the effectiveness of this device has been tested on very selected and strictly controlled young patients over a period of a month from each visit. A longer followup and a more extended population should be investigated.

Many factors influence the periodontal stability in orthodontic patients, such as the duration of orthodontic treatment, forces, biotype and the biology. In conclusion we can state that the use of a mechanical interdental cleaning device combined with manual toothbrush improves self-performed plaque control and gingival health in patients undergoing orthodontic treatment.

Correct oral instructions and the use of a mechanical interdental device could reduce the onset of plaque index, periodontal attachment loss and lesions of the tooth surface. This work is in line with similar studies in the literature, and our results are closely related to those of other authors.

In the future, the possibility to extend this device to non-orthodontic population and use it in daily hygiene should be assessed.

A clearer comprehension of the mechanobiology of orthodontic movements will lead the clinicians to a more enlightened definition of orthodontic treatment plans, especially where a multidisciplinary approach is required.

\section{References}

1. Demling A, Heuer W, Elter C, Heidenblut T, Bach FW, Schwestka-Polly R, et al. Analysis of supra- and subgingival long-term biofilm formation on orthodontic bands. Eur J Orthod 2009;31:202-6.

2. Gorelick L, Geiger AM, Gwinnett AJ. Incidence of white spot formation after bonding and banding. Am J Orthod 1982;81:93-8.

3. Underwood ML, Rawls HR, Zimmerman BF. Clinical evaluation of a fluoride-exchanging resin as an orthodontic adhesive. Am J Orthod Dentofac Orthop 1989;96:939.

4. Cai F, Shen P, Morgan MV, Reynolds EC. Remineralization of enamel subsurface lesions in situ by sugar free lozenges containing casein phosphopeptide-amorphous calcium phosphate. Aust Dent J 2003;48:240-3.

5. Bishara SE, Ostby AW. White spot lesions: formation, prevention, and treatment. Semin Orthod 2008;14:17482

6. O'Reilly MM, Featherstone JD. Demineralization and remineralization around orthodontic appliances: an in vivo study. Am J Orthod Dentofac Orthop 1987;92:3340.

7. Ogaard B, Rolla G, Arends J. Orthodontic appliances and enamel demineralization. Part 1. Lesion development. Am J Orthod Dentofac Orthop 1988;94:68-73.

8. Maxfield BJ, Hamdan AM, Tufekci E, Shroff B, Best AM, Lindauer SJ. Development of white spot lesions during orthodontic treatment: perceptions of patients, parents, orthodontists, and general dentists. Am J Orthod Dentofac Orthop 2012;141:337-44.

9. Kim SH, Choi DS, Jang I, Cha BK, Jost-Brinkmann PG, et al. Microbiologic changes in subgingival plaque before and during the early period of orthodontic treatment. Angle Orthod 2012;82:254-60.

10. Liu Y, Zhang YJ, Wang L, Guo Y, Xiao SQ. Prevalence of Porphyromonas gingivalis Four rag Locus Genotypes in Patients of Orthodontic Gingivitis and Periodontitis. PLoS One 2013;8:e61028.

11. Liu Y, Zhang YJ, Wang L, Guo Y, Xiao SQ. Detection of Fusobacterium Nucleatum and fadA Adhesin Gene in Patients with Orthodontic Gingivitis and Non-Orthodontic Periodontal Inflammation. PLoS One 2014;9:e85280.

12. Tanner AC, Sonis AL, Lif Holgerson P, Starr JR, Nunez $\mathrm{Y}$, Kressirer CA, et al. White-spot lesions and gingivitis microbiotas in orthodontic patients. J Dent Res 2012;91:853-8.

13. Matarese G, Isola G, Ramaglia L, Dalessandri D, Lucchese A, Fabiano F, et al. Periodontal biotype: characteristic, prevalence and dimensions related to dental malocclusion. Minerva Stomatol 2016;65:231-8.

14. de Jager M, Jain V, Schmitt P, DeLaurenti M, Jenkins W, Milleman J, et al. Effect of Philips Sonicare AirFloss on interproximal plaque and gingivitis J Dent Res 2011;90.

15. O'Leary TJ, Drake RB, Naylor JE. The plaque control record. J Periodontol.1972;43:38.

16. Ainamo J, Bay I. Problems and proposals for recording gingivitis and plaque. Int Dent J 1975;25:229-35.

17. Antoun JS, Mei L, Gibbs K, Farella M. Effects of orthodontic treatment on the priodontal tissues. Periodontol 2017;74:140-57.

18. Davies SM, Plonka AB, Fulks BA, Taylor KL, Bashitski J. Consequences of orthodontic treatment on periodontal health: Clinical and microbial effects. Semin Orthod 2014;20:139-49.

19. Krell S, Kaler A, Wei J. Compliance: in vivo study, Inhome use test to assess compliance of Philips Sonicare AirFloss; 2010 [Internet]. Available from: www.sonicareshop.gr/image/data/blog/Compliance.pdf [cited 2018, Feb 20]. 
20. Krell S, Kaler A, Wei J. Preference: In-home use test to evaluate ease of use for Philips Sonicare AirFloss versus Reach string floss and Waterpik Ultra Water Flosser; 2010 [Internet]. Available from: www.sonicareshop.gr/ image/data/blog/Preference.pdf [cited 2018, Feb 20].

21. Feil PH, Grauer JS, Gadbury-Amyot CC, Kula K, McCunniff MD. Intentional use of the Hawthorne effect to improve oral hygiene compliance in orthodontic patients. J Dent Educ 2002;66:1129-35.

22. Burch JG, Lanese R, Ngan P. A two-month study of the effects of oral irrigation and automatic toothbrush use in an adult orthodontic population with fixed appliances. Am J Orthod Dentofacial Orthop 1994;106:121-6.

23. Sharma NC, Lyle DM, Qaqish JG, Galustians J, Schuller R. Effect of a dental water jet with orthodontic tip on plaque and bleeding in adolescent patients with fixed orthodontic appliances. Am J Orthod Dentofacial Orthop 2008;133:565-71; quiz 628 e1-2.

24. de Jager M, Rmaile A, Darch O, Bikker JW. The Effectiveness of Manual versus High-Frequency, High-Amplitude Sonic Powered Toothbrushes for Oral Health: A Meta-Analysis. J Clin Dent 2017;28(1 Spec No A):A1328
25. Walsh M, Heckman B, Leggott P, Armitage G, Robertson PB. Comparison of manual and power toothbrushing, with and without adjunctive oral irrigation, for controlling plaque and gingivitis. J Clin Periodontol 1989;16:419-27.

26. Goyal CR, Lyle DM, 22Qaqish JG, Schuller R. The addition of a water flosser to power toothbrushing: effect on bleeding, gingivitis, and plaque. J Clin Dent 2012;23:5763.

27. Barnes CM, Russell CM, Reinhardt RA, Payne JB, Lyle DM. Comparison of irrigation to floss as an adjunct to toothbrushing: effect on bleeding, gingivitis, and supragingival plaque. J Clin Dent 2005; 16:71-7.

28. Cardaropoli D. Orthodontics for the adult periodontal patient: first or second choice treatment? Prog Orthod 2009; 10:88-96

29. Isola G, Matarese G, Cordasco G, Perillo L, Ramaglia L. Mechanobiology of the tooth movement during the orthodontic treatment: a literature review. Minerva Stomatol 2016;65:299-327.

30. Cavuoti S, Matarese G, Isola G, Abdolreza J, Femiano F, Perillo L. Combined orthodontic-surgical management of a transmigrated mandibular canine: a case report. The Angle Orthodontist 2016;86:681-91.

Conflicts of interest.-Philips Italy has provided the interdental devices used in the present study.

Acknowledgments.-The authors are grateful to prof. Mauro Farella (University of Otago, New Zealand) for his contribution to the first draft of this manuscript; to Dr. Scilla Sparabombe and Dr. Antonio Sabatucci (University of Ancona, Italy) for their kind contribution to patient treatment and for supervising dental hygiene students; to Dr. Anna Guerri and Dr. Elisa Severini for their contribution during the collection of clinical data; and to Mr. Andrew Gray (University of Otago) for performing the statistical analysis. Article first published online: February 13, 2018. - Manuscript accepted: February 8, 2018. - Manuscript revised: Janaury 29, 2018. Manuscript received: September 30, 2017. 